\title{
Combined surgical treatment for missed rupture of triceps tendon associated with avulsion of the ulnar collateral ligament and flexor-pronator muscle mass
}

\author{
Bulent Daglar • Onder M. Delialioglu • \\ Erman Ceyhan - Okyar Altas · \\ Kenan Bayrakci · Ugur Gunel
}

Received: 23 December 2008/ Accepted: 16 March 2009/Published online: 2 April 2009

(C) Springer-Verlag 2009

\begin{abstract}
Triceps tendon ruptures are rare injuries. Coexistence of ipsilateral ulnar collateral ligament injury is even rarer. Here, we describe an unusual combination injury to elbow of a 39-year-old male construction worker consisting of triceps tendon rupture, avulsion of elbow ulnar collateral ligament and flexor pronator muscle origin ipsilaterally. A simultaneous repair and reconstruction of all damaged structures was proposed with individualized postoperative rehabilitation. Return to pre-injury level of activities obtained with this treatment protocol. High degree of suspicion and careful examination were needed to prevent missed diagnosis and prolonged instability which may be inevitable after inappropriate treatment of such injury.
\end{abstract}

Keywords Triceps tendon - Rupture - Elbow · Collateral ligament

\section{Introduction}

Triceps tendon (TT) ruptures are rare injuries. Coexistence of ipsilateral ulnar collateral ligament (UCL) injury is even rarer [1]. To our knowledge, combination of simultaneous rupture of distal TT and avulsion of UCL and flexor-pronator muscle group (FPMG) has not been reported before.

B. Daglar $(\varangle)$. O. M. Delialioglu - E. Ceyhan - O. Altas ·

K. Bayrakci - U. Gunel

4th Clinic of Department of Orthopaedics and Traumatology,

Ankara Numune Training and Research Hospital,

Ankara, Turkey

e-mail: budugul@yahoo.com
The aim of this manuscript is to describe this rare injury and report the result of combined surgical treatment that reconstructs and repairs all damaged structures.

\section{Case report}

A 39-year-old male construction worker was admitted to our department with the complaint of inability to actively extend the right elbow. His history was significant, witha fall from $4 \mathrm{~m} 10$ weeks before the admission. This trauma had initially been treated elsewhere with posterior splint for 3 weeks followed by progressive range of motion exercises. On admission to our institution 10 weeks after the initial trauma, his physical examination revealed a dimple at the posterior aspect of the arm about $4 \mathrm{~cm}$ proximal to the tip of the olecranon (Fig. 1). He was able to fully flex the extended elbow. On the other hand, active extension against gravitational force was not achievable. Neurological examination was normal for both upper extremities. During active flexion of fingers and wrist a painless jerking valgus movement had been observed at the semi-flexed elbow. Valgus stress test for UCL integrity was found positive at the elbow. Plain radiographs showed a soft tissue calcification at the posterior aspect of the distal humerus. Valgus stress radiograph of the elbow revealed severe ulna humeral tilting indicating UCL complex injury (Fig. 1). Magnetic resonance images detected defect in the continuity of the TT and increased intensity around the medial epicondyle (Fig. 2). Combined triceps tendon and UCL rupture was diagnosed. His medical history was inconclusive except for 25 pack/years of smoking. Since the patient had no active elbow extension, a surgical treatment had been advised to repair or reconstruct the damaged structures. 

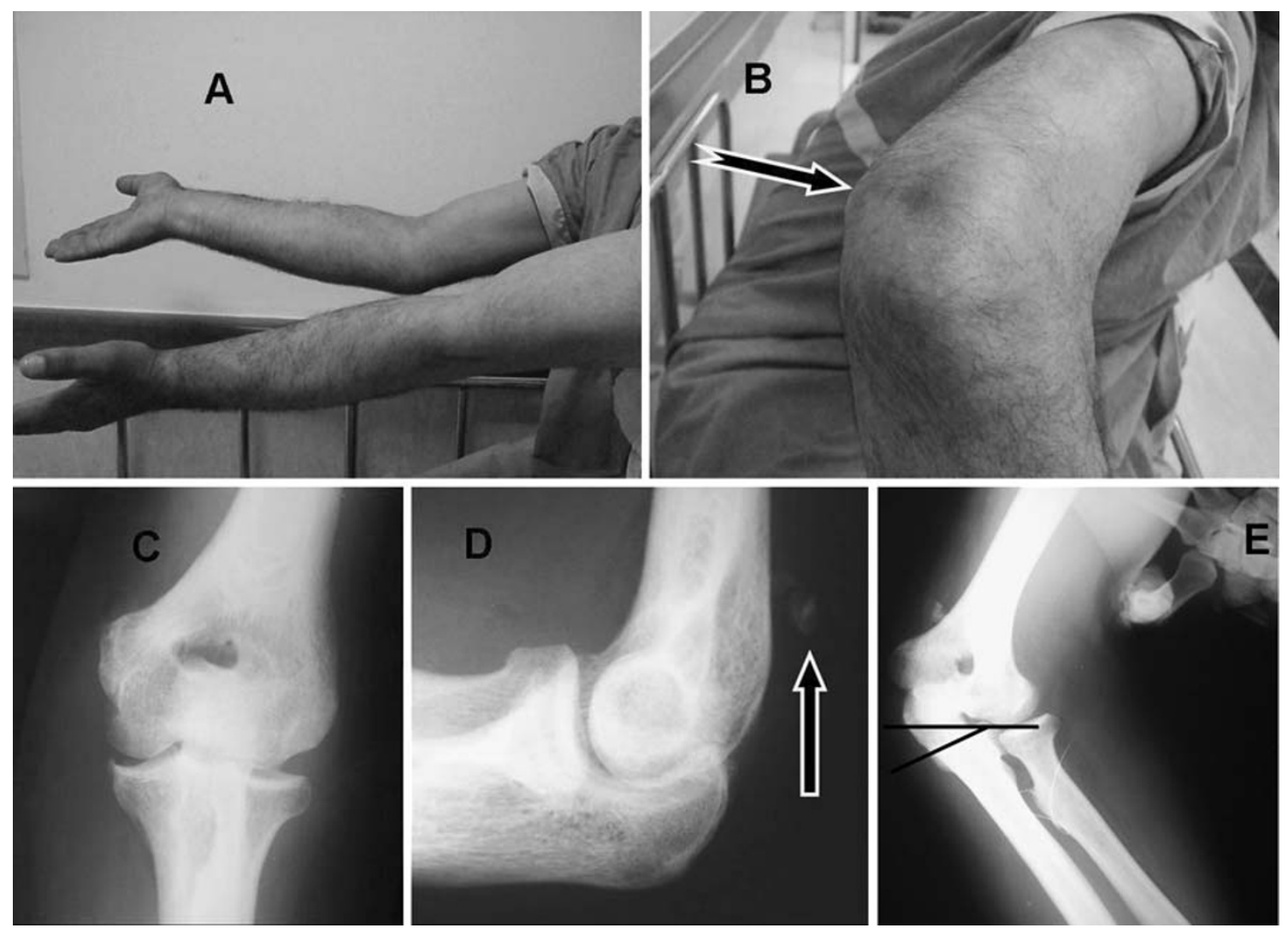

Fig. 1 Preoperative clinical appearance of the patient $(\mathbf{a}, \mathbf{b})$. Note the extension loss at the involved right elbow (a) and dimple (arrow) at the posterior aspect of the elbow (b). Direct radiographs anteroposterior (c) and lateral (d). Lateral radiograph shows 'fleck sign'

\section{Surgical procedure}

Patient was positioned supine and whole right upper extremity was prepared and draped. A sterile tourniquet was applied on upper arm and a $20-\mathrm{cm}$ posterior longitudinal incision was made. Upon opening the subcutaneous layer, the TT was found ruptured and retracted proximally. The gap was filled with dense fibrous healing tissue (Fig. 2). The ulnar nerve was exposed and dissected free from surrounding fibrosis and protected with Penrose drain. Medial epicondyle was observed devoid of any tissue. Flexor-pronator muscle group was found totally avulsed from the medial epicondyle and retracted distally. Ulnar collateral ligament was totally ruptured and remaining parts were attenuated. Triceps tendon was surgically freed from the proximal ulna in continuum with the strong fibrous healing tissue. Since UCL tissue was irreparable, a reconstruction using ipsilateral free palmaris longus tendon graft was performed as described previously [2]. Nonabsorbable sutures in Krackow fashion were used to secure the TT. Four drill holes $1 \mathrm{~cm}$ apart were prepared at the posterior cortex of the proximal ulna for TT fixation. To improve fixation, two $3.5-\mathrm{mm}$ suture anchors were used at (arrow) on posterior aspect of the distal humerus (d). Significant medial opening of the ulno-humeral joint (e) with application of valgus stress (black lines represent humeral and ulnar joint lines)

the tip of the olecranon. Triceps tendon was then secured to the bone while the elbow in $10-20^{\circ}$ of flexion. The ulnar nerve was transferred to the anterior aspect of the elbow submuscularly. Flexor-pronator muscle group was repaired to the medial epicondyle with non-absorbable sutures passing through the bone. Stability and range of motion were tested before the closure over a suction drain. Posterior plaster splint was applied with elbow at $50-60^{\circ}$ flexion.

\section{Postoperative rehabilitation}

Elbow was immobilized for 3 weeks and then gentle active exercises were initiated with hinged elbow brace. Hinge was freed during exercises and locked in a comfortable flexion degree inbetween for another 3 weeks. Six weeks after the operation the patient still used the brace but just for mediolateral stability without limitation of flexion and extension. Strengthening exercises progressed as tolerated by the patient. Brace was discontinued at 3 months postoperatively. Patient returned to active working at 10 months. 


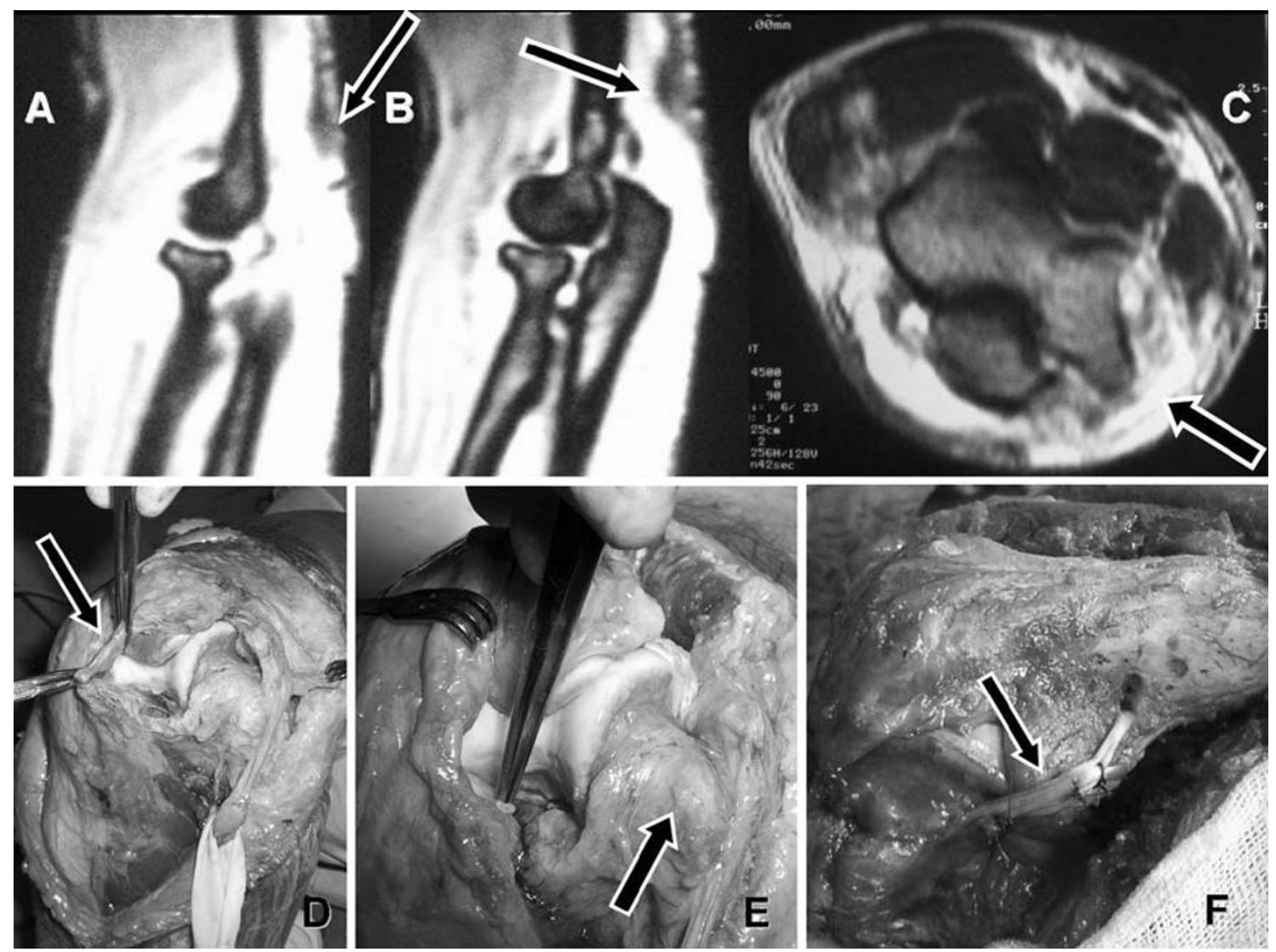

Fig. 2 Preoperative oblique sagital magnetic resonance images showing ruptured triceps tendon (arrow on a) and avulsed bony fragment (arrow on b). Transverse MRI shows increased intensity surrounding the medial epicondyle (c) indicating injury to neighboring structures. Clinical photographs that are taken during the surgery

He was symptom free with stable elbow at the latest follow up. The only limitation was $10^{\circ}$ of extension loss. Otherwise, he regained the full range of elbow motions. Figure 3 shows the clinical photographs of the motions, direct radiographs, and control MRI of the patient.

\section{Discussion}

Triceps tendon partial tears or total ruptures are rare elbow injuries. There are very few case series [3-5] other than isolated case reports that mentioned TT and associated injuries. The usual injury mechanism is a fall on an outstretched hand in which contracted TT eccentrically overloaded with deceleration type impact $[1,3,4,6]$. Direct blow to the posterior aspect of the elbow has also been shown to result in acute ruptures [3]. Although partial tears are well tolerated in activities of daily living, many TT injuries when not diagnosed and treated properly result in prolonged disability [7]. Missed diagnosis complicated the picture in our case as described. show thick fibrous healing tissue (arrow on d). Medial epicondyle is devoid of any tissue (arrow on e) including UCL and FPMG. Reconstruction of UCL (arrow on f) is performed free palmaris longus tendon autograft

Mechanism of injury described that causes rupture of the TT is the common mechanism for many other elbow injuries. So, some other associated ipsilateral elbow injuries may be anticipated for those patients sustained that particular injury pattern. Levy et al. [4] described a syndrome which consists of TT rupture and radial head fracture ipsilaterally. Authors explained the coexistence of this combination and described the mechanism as a decelerating impact after a fall on an outstretched hand followed by the twisting movement of the extended elbow carrying the body weight. The latter creates a great abduction force severing the radial head. Although this mechanism should generate very high moments initially on UCL of the elbow $[8,9]$, the authors did not mention about the injury of this ligament in their report. Coexistence of TT and UCL injuries were clearly described in two cases by Tatebe et al. [1]. In one of their cases, subtle valgus instability was reported after the repair of TT. And in another case, they concluded that gross UCL injury accompanying partial TT tear was resulted from chronic overload since there was a partially intact triceps combined 

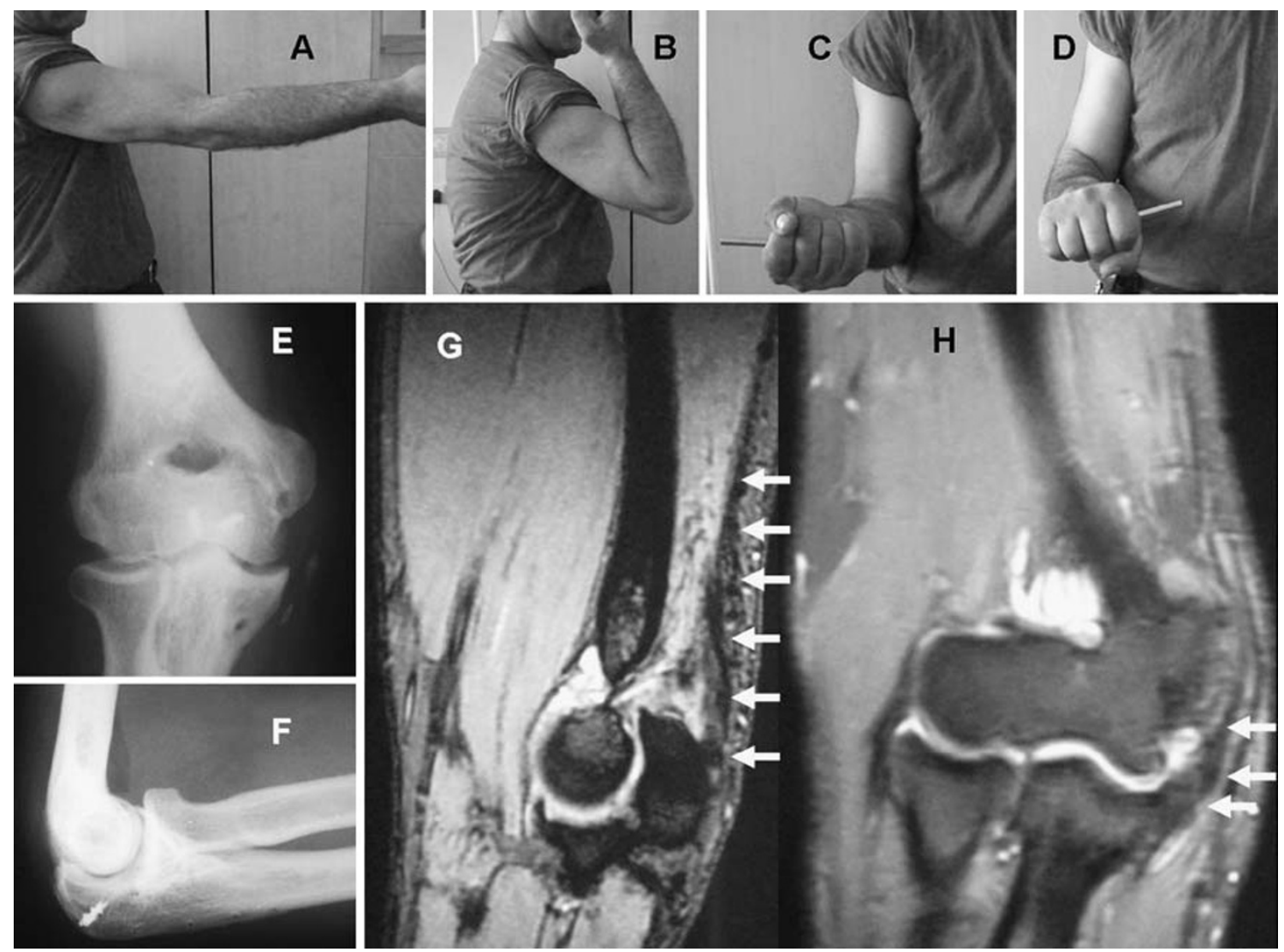

Fig. 3 Clinical photographs of the patient show range of motions of the involved elbow 2 years after surgery (a-d). Direct radiographs show bony tunnels for the UCL reconstruction, small calcifications at the medial side of the joint (e), and holes and metallic anchors used for the TT repair (f). Oblique sagital MRI section shows intact TT (small white arrows on g). Coronal MRI section identifies intact structures at the medial side of the joint (h) with the anconeous muscle, the force vector of the whole elbow extensor is displaced laterally and acts as a valgus stress. These abnormal repetitive valgus torques cause increased stress on UCL complex causing gross valgus instability of the elbow. The presented case in this report had UCL avulsion associated with simultaneous TT total rupture during the initial trauma. Initial conservative treatment did not result in proper healing in either structure leading to incapacitating instability and weakness. Different from the previously presented cases, our patient had additional injury involving flexor-pronator muscle origin which was confirmed during the surgery. To the best of our knowledge, this combination of injury has not previously been described in the literature. Flexor-pronator muscle group, specifically the flexor carpi ulnaris and portions of the flexor digitorum superficialis, lie directly over the anterior bundle of the medial ulnar collateral ligament contributing to the dynamic valgus stability of the elbow [8]. Active protective contraction of wrist and finger flexors together with the valgus stress resulted in avulsion of flexor-pronator common origin. This additional injury may explain our extraordinary clinical examination finding of painless valgus jerking movement of the semi-flexed elbow during active finger or wrist flexion.

Surgery is generally accepted as the best treatment option for total TT ruptures [5, 10, 11]. On the other hand, incomplete tears in which strong lateral fascial connections remain intact and functioning [11, 12] may not cause limitation of daily living activities. These type injuries can be treated conservatively in low demand patients.

Triceps tendon injury itself is a rare condition and combined injuries are even rarer, hence, there is no clinical treatment guideline for these combined injuries. Since he has a high work demand on his elbow, we preferred combined surgery for all damaged structures for our patient to be able to rehabilitate him as early as possible. However, some other authors reported selective repair or reconstruction of associated UCL lesion if necessary following the stability check after the repair of TT [1]. This rare injury combination should be treated according to patient demands and surgeon's experiences. It is our opinion that these combined injuries should be treated surgically at the 
same operation as soon as they are diagnosed. Delay in diagnosis or appropriate treatment renders good results resulting long term disability.

To conclude, here we described a very rare type of elbow injury consisting simultaneous ipsilateral total TT rupture, UCL, and FPMG total avulsion in a young male. Combined surgical repair of all damaged structures 3 months after the index trauma resulted in excellent functional results at early and mid-term follow-up. Therefore, we suggest that all damaged structures should be treated surgically in high demand patients, followed by individualized rehabilitation program. High degree of suspicion and careful examination were needed to prevent prolonged instability which can be inevitable after this type of injuries.

Acknowledgment Authors did not receive any kind of support for this study.

\section{References}

1. Tatebe M, Horii E, Nakamura R (2007) Chronically ruptured triceps tendon with avulsion of the medial collateral ligament: a report of two cases. J Shoulder Elbow Surg 16(1):5-7
2. Stewart AM, Lervick GN, Levine WN (2002) Techniques for ulnar collateral ligament reconstruction in athletes. Curr Opin Orthop 13:298-303

3. Sierra RJ et al (2006) Acute triceps ruptures: case report and retrospective chart review. J Shoulder Elbow Surg 15(1):130-134

4. Levy M, Goldberg I, Meir I (1982) Fracture of the head of the radius with a tear or avulsion of the triceps tendon: a new syndrome? J Bone Joint Surg Br 64B(1):70-72

5. van Riet RP et al (2003) Surgical treatment of distal triceps ruptures. J Bone Joint Surg Am 85-A(10):1961-1967

6. Farrar EL, Lippert FG (1981) Avulsion of the triceps tendon. Clin Orthop Rel Res 161:242-246

7. Sharma SC et al (2005) Missed diagnosis of triceps tendon rupture: a case report and review of literature. J Orthop Surg (Hong Kong) 13:307-309

8. Alcid JG, Ahmad CS, Lee TQ (2004) Elbow anatomy and structural biomechanics. Clin Sports Med 23:503-517

9. Cohen MS, Bruno RJ (2001) The collateral ligaments of the elbow: anatomy and clinical correlation. Clin Orthop Relat Res 383:123-130

10. Pina A, Garcia I, Sabater M (2002) Traumatic avulsion of the triceps brachii. J Orthop Trauma 16:273-276

11. Madsen M et al (2006) Surgical anatomy of the triceps brachii tendon: anatomical study and clinical correlation. Am J Sports Med 34:1839-1843

12. Windisch G, Tesch NP, Grechenig W, Peicha G (2006) The triceps brachii muscle and its insertion on the olecranon. Med Sci Monit 12:290-294 